Cite this: Phys. Chem. Chem. Phys., 2013, 15, 15091

Received 16th April 2013,

Accepted 11th July 2013

DOI: $10.1039 / \mathrm{c} 3 \mathrm{cp} 51621 \mathrm{k}$

www.rsc.org/pccp

\title{
Stability of Si epoxide defects in Si nanowires: a mixed reactive force field/DFT study
}

\author{
Bob Schoeters, ${ }^{\text {ab }}$ Erik C. Neyts, ${ }^{c}$ Umedjon Khalilov, ${ }^{c}$ Geoffrey Pourtois ${ }^{\text {bc }}$ and \\ Bart Partoens ${ }^{a}$
}

\begin{abstract}
Modeling the oxidation process of silicon nanowires through reactive force field based molecular dynamics simulations suggests that the formation of $\mathrm{Si}$ epoxide defects occurs both at the $\mathrm{Si} / \mathrm{SiO}_{x}$ interface and at the nanowire surface, whereas for flat surfaces, this defect is experimentally observed to occur only at the interface as a result of stress. In this paper, we argue that the increasing curvature stabilizes the defect at the nanowire surface, as suggested by our density functional theory calculations. The latter can have important consequences for the opto-electronic properties of thin silicon nanowires, since the epoxide induces an electronic state within the band gap. Removing the epoxide defect by hydrogenation is expected to be possible but becomes increasingly difficult with a reduction of the diameter of the nanowires.
\end{abstract}

\section{Introduction}

Understanding the formation and properties of defective silicon oxide structures at the silica and $\mathrm{Si} / \mathrm{SiO}_{x}$ interfaces is a field of research that has been active for many decades due to the role of Si in semiconductor electronics. Since Moore's law has been driving the reduction of the dimensions of transistors towards nano related dimensions, the effect of interface defects on the electrical performances of transistors has been gaining importance. The use of semiconducting nanowires has already been successfully demonstrated in several nanoelectronics applications such as field-effect-transistors, ${ }^{1}$ sensors ${ }^{2}$ and logic devices. ${ }^{3}$ For a more complete review, see for instance ref. 4 . Therefore, understanding the influence of downscaling the dimensions to the nano regime on the electronic properties of $\mathrm{SiO}$ defect structures in $\mathrm{Si}$ nanowires is essential from a technological point of view. Thin silicon nanowires (SiNW's) and other nanostructures are of interest due to properties which can differ significantly from their bulk state. An important example is the dependence of the SiNW band gap on the quantum confinement. ${ }^{5,6}$ Also, the large surface-to-volume ratio implies that surface effects will soon become very important and might even dominate the properties of these thin nanowires. ${ }^{7}$ In contrast to bulk $\mathrm{Si}$, which has an indirect band gap, thin $\mathrm{Si}$

\footnotetext{
${ }^{a}$ Department of Physics, University of Antwerp, Groenenborgerlaan 171, B-2020 Antwerp, Belgium.E-mail: bob.schoeters@ua.ac.be, bart.partoens@ua.ac.be

${ }^{b}$ IMEC, Kapeldreef 75, B-3001 Leuven, Belgium. E-mail: pourtois@imec.be

${ }^{c}$ Department of Chemistry, University of Antwerp, Universiteitsplein 1, B-2610

Wilrijk-Antwerp, Belgium. E-mail: erik.neyts@ua.ac.be, umedjon.khalilov.ua.ac.be
}

nanowires are expected to have a direct band gap, ${ }^{6}$ which makes them interesting building blocks for opto-electronic applications. Interestingly, no conclusive experimental evidence of direct band gap Si nanowires has been published so far. However, photoluminescence (PL) measurements by Lyons et $a .^{8}$ indicate a significant increase in the PL signal when going from 5 to $4.5 \mathrm{~nm}$ wires, which could be an indication for an indirect to direct band gap transition. Understanding the appearance of defect levels in these band gaps is thus of great importance for possible optical applications.

A common way to obtain very thin SiNW's consists of starting from relatively thick nanowires (of the order of $40 \mathrm{~nm}$ ) grown using a vapor-liquid-solid growth technique, and then using a thermal oxidation process to reduce the diameter. This is typically done by exposing the nanowires to an oxygen ambient at temperatures around $1000{ }^{\circ} \mathrm{C}$. The oxidation process will result in a narrower silicon core surrounded by an amorphous silicon oxide layer. If the thermal oxidation is done at temperatures below $950{ }^{\circ} \mathrm{C}$ the oxidation process becomes self-limiting, meaning that after a certain point in the oxidation process, the oxide layer will not progress further into the Si core. ${ }^{9}$ Once the oxidation process is completed, it is possible to etch the oxide away if required. Depending on the envisioned application, it is also possible to use the obtained core shell structure and exploit the dielectric properties of $\mathrm{SiO}_{x}$ in conjunction with the semiconducting nanowire.

The properties of a thin $\mathrm{Si}$ core surrounded by $\mathrm{SiO}_{x}$ are expected to differ from ideally hydrogen passivated NW's. A considerable effect of stress due to lattice mismatch at the $\mathrm{Si} / \mathrm{SiO}_{x}$ interface can be expected. Ab initio calculations have 
already indicated that the application of stress on SiNW's can have a significant influence on the band gap. ${ }^{10}$ The mismatch at the interface can also induce the formation of defects. For example for $\mathrm{Si}$ nanocrystals with a diameter of $\sim 1.5 \mathrm{~nm}$ surrounded by $\sim 3 \mathrm{~nm}$ of $\mathrm{SiO}_{2}$, the presence of several paramagnetic defects $\left(\mathrm{E}^{\prime}, \mathrm{EX}, \mathrm{P}_{\mathrm{b} 1}\right.$ and $\left.\mathrm{P}_{\mathrm{b}(0)}\right)$ at the interface has already been measured. ${ }^{11}$ The $\mathrm{P}_{\mathrm{b}}$ defects result in states in the band gap that act as recombination centers and are therefore a severe limitation for optical applications. Besides these known defects, it is perfectly possible that other defects are present that are not so easily detected. It was found experimentally, and supported by density functional theory (DFT) calculations and reactive force field based molecular dynamics (MD) simulations, that the formation of Si epoxide (a three membered $\mathrm{Si}-\mathrm{O}-$ Si ring) is an important source of defects at flat silica interfaces. ${ }^{12,13}$ Recent Monte Carlo calculations suggested that this epoxide defect is rather unstable toward structural transformations, particularly when a sizable tensile strain exists along the local surface. ${ }^{10}$ Given the fact that the surface energy (surface strain) in Si nanowires depends on its curvature, it is expected that the diameter of the remaining core will have an impact on the relative stability of the epoxide surface defects. In this paper, we address this issue. The oxidation process of $\mathrm{Si}$ nanowires is investigated using reactive molecular dynamics. Afterwards density functional theory calculations are performed in order to investigate the likeliness of the formation of such epoxide defects. The paper is organized as follows. The computational details of the MD simulations and the DFT calculations are first reviewed. We then investigate the stability of the Si epoxide defect on the surface of a SiNW, and finally discuss its electronic properties in detail.

\section{Computational details}

The MD calculations were based on the reactive Force Field (ReaxFF) developed by van Duin et al. ${ }^{14}$ ReaxFF is based on the bond order/bond distance relationship introduced by Abell. ${ }^{15}$ The total system energy is divided over partial energy terms, related to lone pairs, undercoordination, overcoordination, valence and torsion angles, conjugation, hydrogen bonding, as well as long ranged van der Waals and Coulomb interactions. ${ }^{14}$ These long range interactions are calculated between every pair of atoms within the cutoff length of the interaction, such that the force field not only describes covalent and ionic bonds, but also non-bonded interactions between atoms. Atomic charges are calculated based on the geometry and on the connectivity of the structure using the electron equilibration method (EEM). ${ }^{16}$ In the present work, we employ the parameters developed by Buehler et al. ${ }^{17}$ This parameterization has been successfully applied to different kinds of silica systems, such as the formation of $\mathrm{E}^{\prime}$ centers in amorphous silica, ${ }^{18}$ crack initiation in $\mathrm{Si}^{17,19}$ and the mechanical response of silica in hierarchical structures. $^{20}$ The MD simulations were carried out under NVT conditions, applying the Berendsen thermostat, ${ }^{21}$ with the coupling constant set to 100 fs. The force field interaction length was set to $10 \AA$ and the time step was 0.25 fs. Charges,
Table 1 Number of atoms in the considered SiNW's. All considered SiNW's are (100)-oriented, unless otherwise indictated. The MD simulation of the oxidation process was performed on the wire labeled MD

\begin{tabular}{|c|c|c|c|c|}
\hline Diameter (nm) & Si atoms (\#) & $\mathrm{H}$ atoms $(\#)$ & DFT/LDA & ReaxFF \\
\hline 1.0 & 63 & 60 & 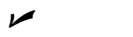 & 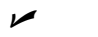 \\
\hline $1.0(110)$ & 64 & 48 & $\nu$ & $x$ \\
\hline $1.0(111)$ & 76 & 60 & & $\times$ \\
\hline 1.5 & 135 & 84 & $\nu$ & $\nu$ \\
\hline 1.8 & 207 & 108 & 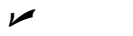 & 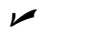 \\
\hline 2.0 & 267 & 132 & $\nu$ & $\nu$ \\
\hline 2.2 & 303 & 132 & 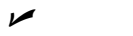 & $\nu$ \\
\hline 4.0 & 1047 & 252 & $x$ & $\nu$ \\
\hline 8.0 & 4107 & 492 & $\times$ & $\nu$ \\
\hline $2.0(\mathrm{MD})$ & 1869 & $\times$ & $\times$ & $\nu$ \\
\hline
\end{tabular}

as calculated using the EEM method, were updated every time step.

DFT calculations, as implemented in the OpenMx code, ${ }^{22-25}$ were performed on (100)-oriented SiNW's, using the local density approximation exchange-correlation energy functional developed by Ceperley and Alder. ${ }^{26}$ A double-valence plus single-polarization orbitals basis set has been used. For single nanowire unit cells a Monkhorts-Pack $k$-point grid of $1 \times 1 \times 6$ was used. To investigate the defect, a supercell of three unit cells in the growth direction is used with an equivalent $1 \times 1 \times$ $2 k$-point grid. The fineness of the numerical grid used for the numerical integrations and for solving the Poisson equation within OpenMx was fixed by using a cutoff energy of 150 Rydberg (2040 eV). The positions of the atoms in the SiNW's were optimized using the method developed by Baker ${ }^{27}$ until the forces were less than $0.005 \mathrm{eV} \AA^{-1}$. Further calculations using the HSE0 ${ }^{28}$ hybrid functional were performed with the Vienna $A b$ Initio Simulation Package (VASP), ${ }^{29,30}$ using the Projector Augmented Wave (PAW) method. ${ }^{31,32}$ Integrations over the Brillioun zone were done using a $1 \times 1 \times 8 k$-point grid. As opposed to OpenMx, VASP uses plane waves as a basis set, whose accuracy has been fixed by using an electronic cutoff of $400 \mathrm{eV}$ resulting in the convergence of the total energy below $10^{-3} \mathrm{eV}$ per atom.

For all calculations, both using DFT and ReaxFF, a vacuum of at least $10 \AA$ was used in the directions perpendicular to the wire axis. Details of all the SiNW's we considered in this work are summarized in Table 1.

\section{Results and discussion}

\subsection{The oxidation of SiNW's}

In order to understand the properties of small diameter SiNW's produced by thermal oxidation, MD simulations were performed using the ReaxFF force field. As previously mentioned in the Introduction, most published experimental work in this field describes a rapid thermal oxidation at temperatures around $1273 \mathrm{~K}$ or more to obtain core-shell structures with a small core, see for example ref. 33 and 34. Therefore we also used this temperature in our simulations. The thermal oxidation process was simulated by adding $\mathrm{O}_{2}$ every 10 ps into a box 
(with periodic boundaries) containing 7 times the smallest unit cell of a crystalline (100)-SiNW with a diameter of $2 \mathrm{~nm}$. During these simulations, we observed the radial growth of $\mathrm{a} \mathrm{SiO}_{x}$ shell with a clearly defined $\mathrm{Si} / \mathrm{SiO}_{x}$ interface, which progressed continuously inward. Some oxygen atoms however diffuse beyond the $\mathrm{Si} / \mathrm{SiO}_{x}$ interface. The oxidation process is not self-limiting since upon a large number of MD steps and an infinite supply of oxygen, the structure will become fully oxidized. This is not unexpected since self-limiting oxidation is experimentally only observed at temperatures below $950{ }^{\circ} \mathrm{C} .{ }^{9}$ A previous MD study ${ }^{35}$ of thermal oxidation with ReaxFF has indicated that the oxidation will be self-limiting for temperatures below $500 \mathrm{~K}, 600 \mathrm{~K}$, $700 \mathrm{~K}$ and $800 \mathrm{~K}$ for SiNW's with a diameter of, respectively, $1.5 \mathrm{~nm}, 2 \mathrm{~nm}, 2.5 \mathrm{~nm}$ and $3 \mathrm{~nm}$. Since we want to investigate a small $\mathrm{Si}$ core surrounded by $\mathrm{SiO}_{x}$, we considered wires where the thermal oxidation process is not completed. In order to analyze the structural properties, we stopped the MD simulation at a selected time. A rapid quench to $0 \mathrm{~K}$ was then simulated by performing a relaxation using a conjugate gradient algorithm. Two stages from these MD simulations are illustrated in Fig. 1, corresponding to approximately a $\mathrm{SiO}_{0.25}$ and $\mathrm{SiO}_{0.5}$ oxidation stage.

The main building blocks for $\mathrm{SiO}_{2}$ are based on $\mathrm{SiO}_{4}$ tetrahedrons. In crystalline $\mathrm{SiO}_{2}$, the latter forms an ordered network that gets disordered upon amorphization. Clearly all the bonds present in $\mathrm{SiO}_{2}$ are $\mathrm{Si}-\mathrm{O}$ ones settled within $\mathrm{Si}-\mathrm{O}-\mathrm{Si}$ bridge bonds. The angle between these $\mathrm{Si}-\mathrm{O}-\mathrm{Si}$ bridge bonds is very flexible, which results in structures that vary significantly but that are still based on the same building blocks. This is also what we obtained in our fully oxidized wires. In the final structure, all the Si-Si bonds were broken and essentially only the $\mathrm{Si}-\mathrm{O}$ ones remained. However, if we now focus on the clearly observable $\mathrm{Si} / \mathrm{SiO}_{x}$ interface in our simulated nanowires, new Si-O-Si features are observed, i.e. Si epoxide defects which are three membered $\mathrm{Si}-\mathrm{O}-\mathrm{Si}$ rings. In Fig. 2(a) we focus the $\mathrm{Si} / \mathrm{SiO}_{x}$ interface of our $\mathrm{SiO}_{0.5}$ nanowire, here we see both $\mathrm{Si}-\mathrm{O}-\mathrm{Si}$ bridge bonds and epoxide defects. Furthermore, this epoxide defect is also abundantly present at the $\mathrm{SiO}_{x}$ surface (Fig. 2(b)).
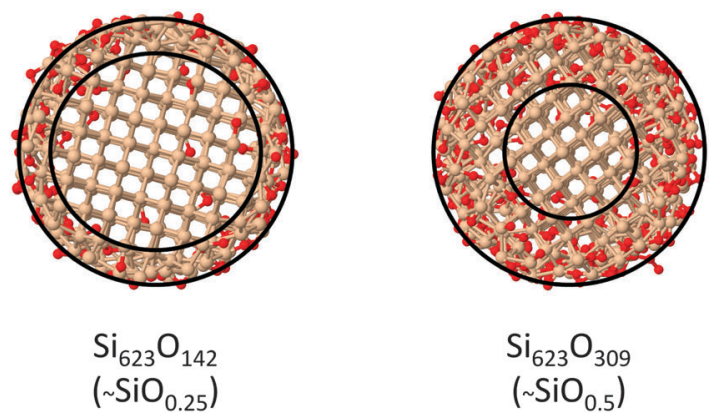

Fig. 1 Illustration of two steps extracted from the MD simulation that correspond to approximate $\mathrm{SiO}_{0.25}$ and $\mathrm{SiO}_{0.5}$ stoichiometry obtained after rapid quenching to $0 \mathrm{~K}$. A continuous progression of the $\mathrm{Si} / \mathrm{SiO}_{x}$ is clearly visible with some $\mathrm{O}$ atoms diffusing beyond the interface. The $\mathrm{SiO}_{x}$ region is indicated by continuous circles.

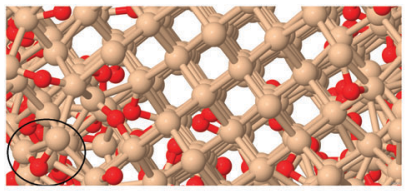

(a) (b)

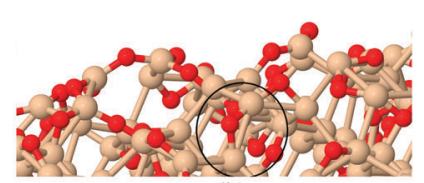

Fig. 2 Si epoxide defects in $\mathrm{SiO}_{0.5}$ at the $\mathrm{Si} / \mathrm{SiO}_{x}$ interface (a) and at the $\mathrm{SiO}_{x}$ surface (b). In both figures, one Si epoxide defect is encircled but many more are present.

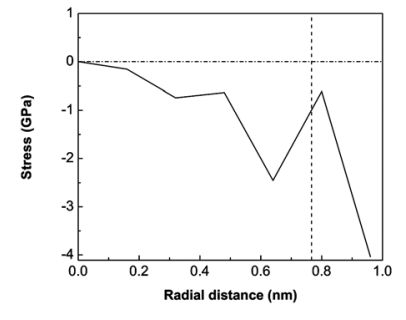

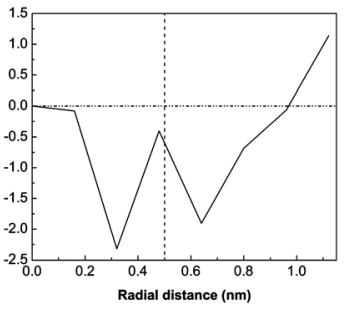

Fig. 3 The $r r$ component of the stress for the $\mathrm{SiO}_{0.25}$ (a) and $\mathrm{SiO}_{0.5}$ (b) wire plotted along the radial distance. The dashed vertical line corresponds to the Si/ $\mathrm{SiO}_{x}$ interface.

A possible origin of the $\mathrm{Si}$ epoxide defects at the $\mathrm{Si} / \mathrm{Si}_{x}$ interface is the stress generated by the lattice mismatch between $\mathrm{Si}$ and $\mathrm{SiO}_{x}$. To quantify this, we calculated the Cauchy stress in the nanowires ${ }^{36}$ after rapid quench to $0 \mathrm{~K}$. Because we consider the system after rapid quench to $0 \mathrm{~K}$, we avoid the kinetic energy contribution to the stress, which could be problematic. ${ }^{37}$ In Fig. 3 we plot the $r r$ component of the stress along the radial direction of the nanowire. This reveals that a tensile stress of approximately $2 \mathrm{GPa}$ occurs around the $\mathrm{Si} / \mathrm{SiO}_{2}$ interface, which is also observed in previous simulations. ${ }^{38}$ The fact that the stress is tensile is in agreement with the thermal oxidation not being self-limiting. If the $r r$ component of the stress is compressive the reaction rate of the oxidation will lowered causing the oxidation to slow down or even stop, ${ }^{38,39}$ this being the cause of the so called self-limiting oxidation. Tensile stress on the other hand will enhance the oxidation. As mentioned before, self-limiting oxidation is only expected at low temperatures. Here we simulate a rapid thermal oxidation at high temperature. Therefore the computed stresses are in accordance with the observed behavior of the oxidation process in our simulation.

The number of epoxide defects changes during the oxidation process. In the first steps of the simulation, almost all oxygen atoms at the nanowire surface form epoxide defects. As the oxidation process progresses, more of the oxygen atoms will settle in the more stable non-triangular bridge bonds. Fig. 4(a) depicts the number of $\mathrm{Si}$ epoxide defects and $\mathrm{Si}-\mathrm{O}-\mathrm{Si}$ bridge bonds in our unit cell at the nanowire surface as a function of the oxidation grade, where we define the surface as the two outer atomic layers. Fig. 4(b) also depicts this evolution but for the full wire. As mentioned before after full oxidation, we obtain a structure containing only $\mathrm{Si}-\mathrm{O}-\mathrm{Si}$ features (approximately $\mathrm{SiO}_{2}$ ). So clearly all epoxide defects are gone. But unless 


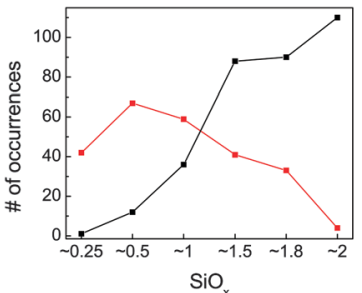

(a)

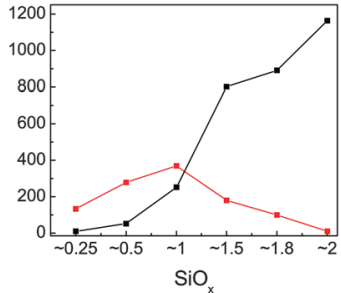

(b)

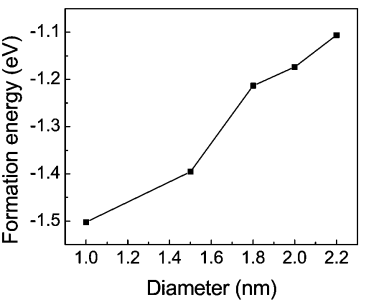

(a)

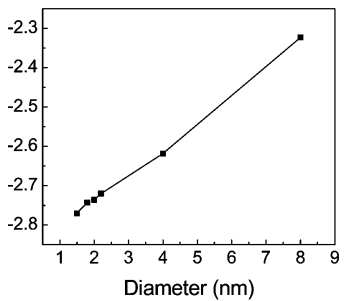

(b)
Fig. 4 Comparison between the number of Si epoxide defects (red) and the $\mathrm{Si-O}-\mathrm{Si}$ bridge bonds (black) present in our unit cell (a) at the nanowire surface and $(b)$ in the whole wire as a function of the oxidation level.

the nanowire is completely oxidized a relatively large amount of Si epoxide will remain at the surface.

It is also interesting to note that this Si epoxide defect is also visible in Fig. 1 and 2 of the work of Bondi et al., ${ }^{40}$ in which a combination of Monte Carlo and DFT calculations were performed to study oxidized SiNW's. However it was not discussed in this reference.

\subsection{Stability of the epoxide defect}

Due to the curvature of cylindrical nanowires, the surface will also face a large amount of stress that might induce the defect. In order to understand better this Si epoxide structure and the effect of the surface curvature on its relative stability, we investigated the evolution of the formation energy of a single defect on hydrogen passivated (100)-SiNW's of different diameters using DFT calculations based on LDA. On these hydrogen passivated wires, we replaced two hydrogen atoms with a single oxygen atom (Fig. 5) and after relaxation we computed its formation energy as:

$$
E_{\mathrm{f}}=E(\operatorname{SiNW}+\text { defect })-E(\operatorname{SiNW})+2 \mu_{\mathrm{H}}-\mu_{\mathrm{O}}
$$

with $\mu$ being the chemical potential of, respectively, hydrogen $\left(\mu_{\mathrm{H}}\right)$ and oxygen $\left(\mu_{\mathrm{O}}\right)$. Half of the total energy of, respectively, $\mathrm{H}_{2}$ and of the triplet state of $\mathrm{O}_{2}$ has been used as the chemical potential. To minimize the self-interaction of the defect due to the periodic conditions, a supercell of three times the smallest unit cell in the growth direction was used, which ensures the convergence of the $E_{\mathrm{f}}$ of the neutral Si epoxide defect. The formation energy was then evaluated for different wire diameters going from 1 to $2.2 \mathrm{~nm}$. The results are summarized in Fig. 6(a). Interestingly, the formation energy of the defect is

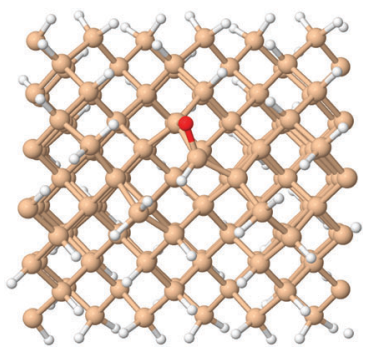

Fig. 5 Defect on a $1.5 \mathrm{~nm}$ pristine wire. The Si atoms are depicted as pale orange spheres, hydrogen as white ones and oxygen red.
Fig. 6 Evolution of the formation energy with respect to the diameter of the nanowire, with DFT (a) and ReaxFF (b).

reduced for large wires, while for smaller ones, it is enhanced, suggesting that the increasing curvature at smaller diameters helps stabilizing the epoxide defect.

Unfortunately, it is computationally very demanding to tackle large diameter wires using ab initio calculations due to the large number of atoms involved. Therefore, we also used ReaxFF to evaluate the formation energy of the defect on wires, as shown in Fig. 6(b). Note that the absolute values of the formation energy obtained using ReaxFF are not the same as those obtained using our DFT calculations. We attribute this difference to the fact that the fitting of the ReaxFF parameterization was done using different DFT functionals than the one we used in this work. Indeed different functionals can result in a significantly different formation energy, see for example ref. 41 Nevertheless, the ReaxFF energies show qualitatively the same trend, the defect becomes less and less stable with an increasing diameter. The largest wire has a diameter of $8 \mathrm{~nm}$ which still amounts to a considerable curvature and saturation is therefore not achieved yet. Note that the limit of $d \rightarrow \infty$ does not correspond with the epoxide defect on a flat surface. Indeed the coordination of the hydrogen atoms that passivate dangling bonds on a facet of flat $\mathrm{Si}$ is not the same as that on the corresponding facet of a curved cylindrical nanowire, hence resulting in a slightly different structure around the defect. Therefore the comparison between flat and curved Si surfaces is not straightforward.

\subsection{Electronic structure}

Now, we turn to the electronic properties of this Si epoxide defect on a SiNW and compare the density of states (DOS) of the wire with and without defect (Fig. 7(a)) obtained using DFT calculations. The figure clearly shows that the defect induces electronic states within the band gap at about $0.2 \mathrm{eV}$ with respect to the top of the valence band.

Since the formation energy of the defect changes for different diameters it would not be surprising if the the electronic properties are also impacted. Fig. 8(a) illustrates the evolution of the position of the occupied defect level compared to the conduction band minimum (CBM) and valence band maximum (VBM) as a function of the diameter. With an increase of the diameters and due to confinement effects, the defect level is gradually shifted towards the valence band. Although the amplitude of this shift is reduced for larger diameter, the 


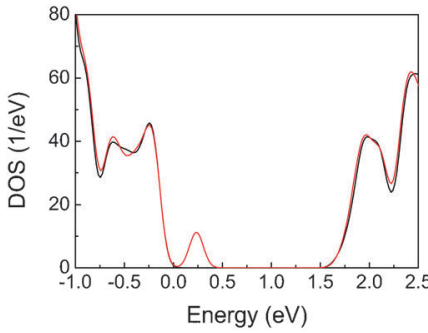

(a)

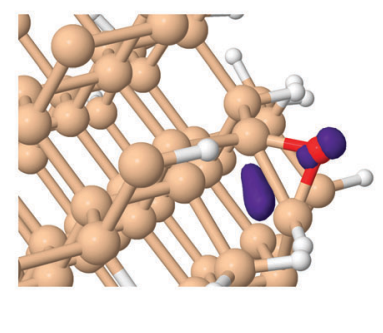

(b)
Fig. 7 (a) Density of states computed for a $1.5 \mathrm{~nm}$ Si nanowire in the presence (red) and the absence (black) of the epoxide defect. Zero is set to the valence band maximum. (b) Bloch state integrated over the energy window associated with the Si epoxide defect for a $1.5 \mathrm{~nm}$ nanowire.

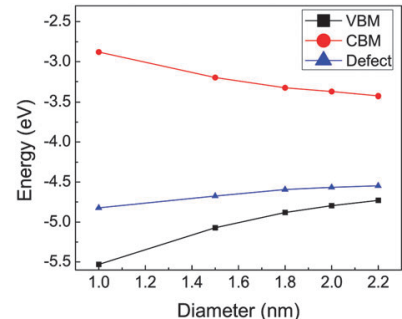

(a)

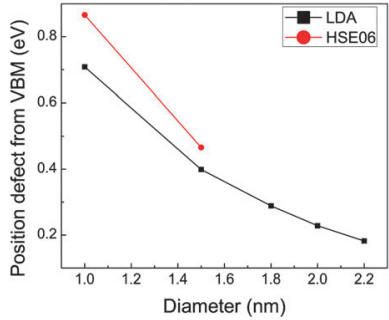

(b)
Fig. 8 (a) Energy of the electronic states induced by the presence of a Si epoxide defect compared to the VBM and CBM as a function of the diameter, with the energies defined relative to the vacuum. (b) Comparison of the position of the energy of the electronic states induced by the Si epoxide defect relative to VBM calculated with LDA (black) and HSE06 (red).

extrapolation suggests that the defect level disappears into the valence band for diameters of $\sim 3.5 \mathrm{~nm}$ and larger.

It is well known that the LDA approximation suffers from a band gap underestimation problem. To improve the description of the electronic properties we also performed HSE06 hybrid functional calculations for the two smallest wires. For bulk silicon HSE06 is known to give a good description of the electronic properties. ${ }^{42}$ The results for oxides are also a considerable improvement over LDA. ${ }^{42,43}$ In Fig. 8(b), the location of the defect level is shown relative to the valence band maximum, for both LDA and HSE06. Although the absolute value for the band gap calculated using the HSE06 functional differs by about $1 \mathrm{eV}$, the qualitative evolution of the defect level obtained with LDA remains valid. The nature of the state in the electronic gap is revealed by the spatial distribution of the Bloch states integrated over the energy window associated with the defect. Fig. 7(b) illustrates that there is a contribution from the oxygen atom but also from the strained bond between the underlying Si atoms. This confirms that the defect is caused by the strain that is generated at the surface as a result of the curvature.

\subsection{Removal of the epoxide defect by hydrogenation}

Given the presence of this defective source in the electronic gap and its influence on the electronic properties, it is of prime importance to evaluate the possibility to 'passivate' it. We

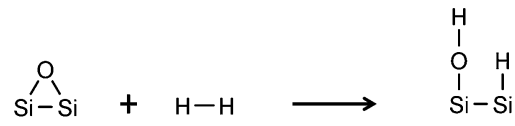

Fig. 9 Schematic drawing of the hydrogenation process of the epoxide defect.

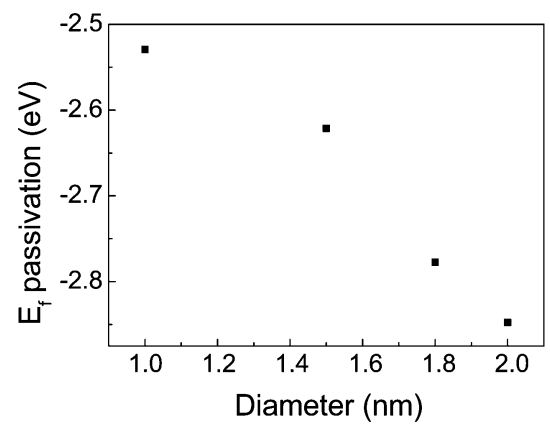

Fig. 10 Enthalpy of reaction computed for the passivation of an epoxide defect in the presence of $\mathrm{H}_{2}$.

hence investigated its reactivity with hydrogen, a common passivation source used in the microelectronic industry (Fig. 9).

We now define the enthalpy of reaction of this hydrogenation as:

$$
E_{\mathrm{f}}=E(\operatorname{SiNW}+\mathrm{OH} / \mathrm{H})-E(\operatorname{SiNW}+\text { defect })-2 \mu_{\mathrm{H}}
$$

with $\mu_{\mathrm{H}}$ being the chemical potential of hydrogen. As indicated in Fig. 10, the enthalpy of reaction obtained within the LDA is exothermic. Interestingly, the dependence of the relative stability of the defect with respect to the diameter of the nanowire is reflected into the enthalpy of reaction dependence of the diameter, with a suppressed exothermicity for smaller nanowire diameters. The exothermicity suggests that the reaction is favorable, however there remains an activation energy that the $\mathrm{H}_{2}$ has to overcome before it can bind with the defect. In order to evaluate the minimum energy path for the hydrogen passivation of this epoxide defect, which gives us the activation energy, we used the nudged elastic band method. ${ }^{44}$ This pathway was calculated for the hydrogenation of a $1.0 \mathrm{~nm}$ wire. The total energy of the system after hydrogenation is $2.5 \mathrm{eV}$ lower than before, but first a barrier of $\sim 0.5 \mathrm{eV}$ has to be overcome. This activation energy is of the same order as the barrier that has to be overcome to passivate dangling bonds on a $\operatorname{Si}(100)-2 \times 1$ surface. ${ }^{45}$ The hydrogenation results in the removal of the state in the band gap.

\subsection{Influence of growth orientations}

All presented results were for (100)-oriented SiNW's. We also investigated the impact of the relative orientation of the $\mathrm{Si}$ nanowire on the stability of the epoxide defect and its electronic signature in the band gap, within the LDA approximation. Apart from the (100)-oriented SiNW's, we also focused on (110) and (111) SiNW's, and evaluated the differences for nanowires with a diameter of $1.0 \mathrm{~nm}$. The formation energies of a single epoxide defect are, respectively, $-1.50 \mathrm{eV},-1.24 \mathrm{eV}$ and $-1.39 \mathrm{eV}$. This indicates that the defect is most stable on the surface of (100)-oriented nanowires, but the difference in formation 


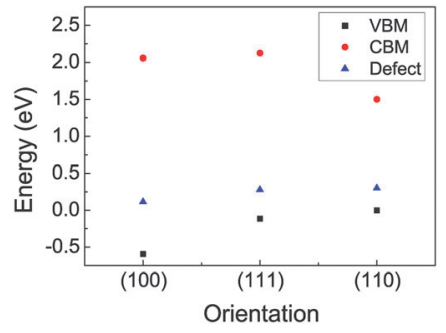

Fig. 11 Position of the defect level compared to VBM and CBM for three differently oriented $1.0 \mathrm{~nm}$ wires, calculated using the LDA functionals. The alignment was done compared to a vacuum and the VBM of the (110)-nanowire was set to zero.

energy is not very large. Although the Si nanowire band gap is different for the different orientations, the defect induces an electronic signature within the electronic gap in all three cases. This is illustrated in Fig. 11, where the position of the defect is depicted for $1.0 \mathrm{~nm}$ nanowires of the three different orientations. Note that the absolute position of the defect, compared to the vacuum, is the same for the three different orientations. However, due to the different positions of the VBM and CBM, the defect level is situated differently within the band gap for the different orientations.

\section{Conclusions}

In this paper, we present a mixed reactive force field/DFT study of the properties of an epoxide defect in Si nanowires. MD simulations of the oxidation process suggest that this defect is present both at the $\mathrm{Si} / \mathrm{SiO}_{x}$ interface and at the nanowire surface, as opposed to flat surfaces where it has been reported to occur upon the presence of a stress generated by a lattice mismatch. The formation energy of these Si epoxide defects at the surface of Si nanowires drops for reduced diameters of the nanowires, which indicates an increase in stability. We attribute this increase in stability to the curvature of the wire, which generates strained bonds at the surface. The epoxide defect has an electronic signature within the band gap, which can be an issue for microelectronic and optical applications. A hydrogenation treatment of the defect at the surface will remove the state in the band gap but an activation energy of the order of $0.5 \mathrm{eV}$ must be overcome.

\section{Acknowledgements}

BS gratefully acknowledges financial support of the IWT, Institute for the Promotion of Innovation by Science and Technology in Flanders, via the SBO project "SilaSol". This work was carried out using the Turing HPC infrastructure at the CalcUA core facility of the Universiteit Antwerpen, a division of the Flemish Supercomputer Center VSC, funded by the Hercules Foundation, the Flemish government and the Universiteit Antwerpen.

\section{References}

1 Y. Cui, Z. Zhong, D. Wang, W. U. Wang and C. M. Lieber, Nano Lett., 2003, 3, 149-152.
2 Y. Cui, Q. Wei, H. Park and C. M. Lieber, Science, 2001, 293, 1289-1292.

3 Y. Huang, X. Duan, Y. Cui, L. J. Lauhon, K.-H. Kim and C. M. Lieber, Science, 2001, 294, 1313-1317.

4 W. Lu and C. M. Lieber, J. Phys. D: Appl. Phys., 2006, 39, R387.

5 D. D. D. Ma, C. S. Lee, F. C. K. Au, S. Y. Tong and S. T. Lee, Science, 2003, 299, 1874-1877.

6 X. Zhao, C. M. Wei, L. Yang and M. Y. Chou, Phys. Rev. Lett., 2004, 92, 236805.

7 B. Lee and R. E. Rudd, Phys. Rev. B: Condens. Matter Mater. Phys., 2007, 75, 041305.

8 D. M. Lyons, K. M. Ryan, M. A. Morris and J. D. Holmes, Nano Lett., 2002, 2, 811-816.

9 H. Liu, D. Biegelsen, F. Ponce, N. Johnson and R. Pease, Appl. Phys. Lett., 1994, 64, 1383.

10 C.-L. Kuo and G. S. Hwang, Phys. Rev. Lett., 2006, 97, 066101. 11 S. Godefroo, M. Hayne, M. Jivanescu, A. Stesmans, M. Zacharias, O. I. Lebedev and M. V. V. Van Tendeloo, Nat. Nano, 2008, 3, 174-178.

12 B. B. Stefanov, A. B. Gurevich, M. K. Weldon, K. Raghavachari and Y. J. Chabal, Phys. Rev. Lett., 1998, 81, 3908-3911.

13 U. Khalilov, G. Pourtois, A. C. T. van Duin and E. C. Neyts, J. Phys. Chem. C, 2012, 116, 8649-8656.

14 A. C. T. van Duin, S. Dasgupta, F. Lorant and W. A. Goddard, J. Phys. Chem. A, 2001, 105, 9396-9409.

15 G. C. Abell, Phys. Rev. B: Condens. Matter Mater. Phys., 1985, 31, 6184-6196.

16 W. J. Mortier, S. K. Ghosh and S. Shankar, J. Am. Chem. Soc., 1986, 108, 4315-4320.

17 M. J. Buehler, A. C. T. van Duin and W. A. Goddard, Phys. Rev. Lett., 2006, 96, 095505.

18 N. L. Anderson, R. P. Vedula, P. A. Schultz, R. M. Van Ginhoven and A. Strachan, Phys. Rev. Lett., 2011, 106, 206402.

19 D. Sen, C. Thaulow, S. V. Schieffer, A. Cohen and M. J. Buehler, Phys. Rev. Lett., 2010, 104, 235502.

20 A. Garcia, D. Sen and M. Buehler, Metall. Mater. Trans. A, 2011, 42, 3889-3897.

21 H. J. C. Berendsen, J. P. M. Postma, W. F. van Gunsteren, A. DiNola and J. R. Haak, J. Chem. Phys., 1984, 81, 3684-3690.

22 T. Ozaki, Phys. Rev. B: Condens. Matter Mater. Phys., 2003, 67, 155108.

23 T. Ozaki and H. Kino, Phys. Rev. B: Condens. Matter Mater. Phys., 2004, 69, 195113.

24 T. Ozaki and H. Kino, Phys. Rev. B: Condens. Matter Mater. Phys., 2005, 72, 045121.

25 http://www.openmx-square.org.

26 D. M. Ceperley and B. J. Alder, Phys. Rev. Lett., 1980, 45, 566-569.

27 J. Baker, J. Comput. Chem., 1986, 7, 385-395.

28 J. Heyd, G. E. Scuseria and M. Ernzerhof, J. Chem. Phys., 2003, 118, 8207-8215, DOI: 10.1063/1.1564060.

29 G. Kresse and J. Furthmüller, Comput. Mater. Sci., 1996, 6, 15-50. 
30 G. Kresse and J. Furthmüller, Phys. Rev. B: Condens. Matter Mater. Phys., 1996, 54, 11169-11186.

31 P. E. Blöchl, Phys. Rev. B: Condens. Matter Mater. Phys., 1994, 50, 17953-17979.

32 G. Kresse and D. Joubert, Phys. Rev. B: Condens. Matter Mater. Phys., 1999, 59, 1758-1775.

33 R. Ulbricht, R. Kurstjens and M. Bonn, Nano Lett., 2012, 12, 3821-3827.

34 N. Fukata, T. Oshima, K. Murakami, T. Kizuka, T. Tsurui and S. Ito, Appl. Phys. Lett., 2005, 86, 213112.

35 U. Khalilov, G. Pourtois, A. C. T. v. Duin and E. C. Neyts, Chem. Mater., 2012, 24, 2141-2147.

36 I. Daruka, A.-L. Barabási, S. J. Zhou, T. C. Germann, P. S. Lomdahl and A. R. Bishop, Phys. Rev. B: Condens. Matter Mater. Phys., 1999, 60, R2150-R2153.

37 M. Zhou, Proc. R. Soc. London, Ser. A, 2003, 459, 2347-2392.
38 U. Khalilov, G. Pourtois, A. Bogaerts, A. C. T. van Duin and E. C. Neyts, Nanoscale, 2013, 5, 719-725.

39 D.-B. Kao, J. P. McVittie, W. Nix and K. Saraswat, IEEE Trans. Electron Devices, 1988, 35, 25-37.

40 R. J. Bondi, S. Lee and G. S. Hwang, ACS Nano, 2011, 5, 1713-1723.

41 W.-K. Leung, R. J. Needs, G. Rajagopal, S. Itoh and S. Ihara, Phys. Rev. Lett., 1999, 83, 2351-2354.

42 S. Park, B. Lee, S. H. Jeon and S. Han, Curr. Appl. Phys., 2011, 11, S337-S340.

43 M. A. L. Marques, J. Vidal, M. J. T. Oliveira, L. Reining and S. Botti, Phys. Rev. B: Condens. Matter Mater. Phys., 2011, 83, 035119.

44 G. Henkelman and H. Jonsson, J. Chem. Phys., 2000, 113, 9978-9985.

45 D. R. Alfonso and K. D. Jordan, J. Comput. Chem., 2003, 24, 990-996. 